\title{
A Pilot Study of a Medical Cannabis - Opioid Reduction Program
}

\author{
Kevin $\operatorname{Rod}^{1,2}$ \\ ${ }^{1}$ Department of Family and Community Medicine, Faculty of Medicine, University of Toronto, Toronto, Canada \\ ${ }^{2}$ Toronto Poly Clinic Multi-Disciplinary Pain Management Centres, Toronto, Canada
}

\section{Email address:}

krod@tpclinic.com

\section{To cite this article:}

Kevin Rod. A Pilot Study of a Medical Cannabis - Opioid Reduction Program. American Journal of Psychiatry and Neuroscience. Vol. 7, No. 3, 2019, pp. 74-77. doi: 10.11648/j.ajpn.20190703.14

Received: December 20, 2018; Accepted: September 6, 2019; Published: September 20, 2019

\begin{abstract}
Many chronic pain patients are prescribed opioids at doses exceeding the current Guideline. Tapering the dose can be difficult, as patients fear a return to a state of overwhelming pain. Several factors can increase the likelihood of success: the patient's readiness for change, psychological support, pharmacological support and careful monitoring. This pilot study addressed these four factors. Six hundred patients took part. Each was taking daily opioid doses ranging from 90-240 mg morphine equivalent dose (MED). All indicated they were prepared to reduce their opioid dose. Over a six-month period, opioid doses were tapered according to individual needs, usually 10\% every 1-2 weeks. Psychological support was provided through a freely available web-based mental health and wellness tool. Medical cannabis provided pharmacological support at the rate of $0.5 \mathrm{~g} /$ day for each $10 \%$ reduction in opioid dose, as needed. Physicians monitored patients regularly according to each patient's needs. After 6 months, 156 patients (26\%) had ceased taking opioids. An additional 329 patients (55\%) had reduced their opioid use by an average of 30\%. One hundred fourteen patients (19\%) neither increased nor decreased their opioid use. The one patient whose opioid dose was increased had poorly controlled pain and an aggravated pain condition. The success of this medical cannabis - opioid reduction program in a large proportion of patients is grounds for further investigation.
\end{abstract}

Keywords: Opioids, Pain Management, Cannabis, Tapering

\section{Introduction}

Opioids are regularly prescribed to help chronic pain patients manage their condition and regain control of their lives. The 2017 Canadian Guideline for Opioids for Chronic Non-Cancer Pain recommends a maximum daily dose (watchful dose) equivalent to $90 \mathrm{mg}$ morphine (MED) [1], yet higher doses are often used. The recent steep rise in opioid-related deaths, many tied to prescribed medications [2], causes great concern. As a result, physicians are considering tapering the opioid regimens for many of their chronic pain patients, using a patient-centered approach. Tapering requires as much care as prescribing opioids.

Patients' greatest fear when tapering opioids is returning to their previous state of overwhelming chronic pain. Effectively communicating the expected improved function without worsening pain can be reassuring, but patients must be ready for such an important change in their lives. Prochaska's Transtheoretical Model of the Stages of Change [3] provides a framework for assessing the patient's readiness for change [4]. A positive relationship and strong therapeutic alliance of patient and physician is paramount.

Tapering opioids requires a concrete plan to which the patient can commit. Taper rates must be appropriate for each patient, inversely related to the duration of opioid treatment [5], and maintaining scheduled dosing as long as possible [6]. A weekly decrease of $10 \%$ is usually safe and effective [5]. Providing both pharmacological and psychological support can improve outcomes of tapering opioids [5].

Cannabis shows promise as a pharmacological support through tapering, helping to manage pain [7]. In a recent study, chronic pain patients using medical cannabis reported "significant decreases in medication side effects that affected their daily functioning (including opioids), decreases in total number of medications being taken, and improvements in 
quality of life" [8]. The mean decrease in opioid use for these patients was $64 \%$, without any structured tapering program. In another study, chronic pain patients using cannabis for pain relief reported $70 \%$ pain relief (where $100 \%$ is no pain) from marijuana, as opposed to $50 \%$ from their other medications (including opioids) [9]. In a randomized controlled trial, patients inhaling $25 \mathrm{mg}$ of dried cannabis with $9.4 \%$ tetrahydrocannabinol (THC) three times daily reported reduced pain intensity and improved sleep [10]. Cannabis enhances pain relief in patients co-medicated with opioids [11]. Since Canada authorized the use of medical cannabis, use of opioids has declined by $16 \%$ and benzodiazepines by almost $30 \%$ [12]. Cannabis is amenable to self-titration, not only giving patients a better sense of control in their life, but also enabling reduced drug use.

Psychological therapies form part of multi-disciplinary pain management programs. The most common are Cognitive Behavior Therapy (CBT), Acceptance and Commitment Therapy (ACT), mindfulness and relaxation. Brief meditation training can decrease patients' pain ratings and sensitivity to pain [13].

Regular monitoring, scheduled according to each patient's needs and level of risk, is critical.

\section{Methods}

The Research Ethics Board of IRB Services approved the protocol for this pilot study of a Medical Cannabis - Opioid Reduction Program (MCORP). Six hundred adult chronic pain patients at Toronto Poly Clinic provided written informed consent to participate. Each participant was taking daily opioid doses ranging from 90 to $240 \mathrm{mg}$ morphine equivalent (average 120mg MED). (Table 1) All indicated they were prepared to reduce their opioid use.

Table 1. Opioid doses of participants at outset of a Medical Cannabis Opioid Reduction Program.

\begin{tabular}{ll}
\hline \# patients & Original daily opioid dose (MED) \\
\hline 255 & $90 \mathrm{mg}$ \\
150 & $120 \mathrm{mg}$ \\
100 & $150 \mathrm{mg}$ \\
50 & $180 \mathrm{mg}$ \\
20 & $200 \mathrm{mg}$ \\
15 & $220 \mathrm{mg}$ \\
10 & $240 \mathrm{mg}$ \\
Total: 600 & $120 \mathrm{mg}$ (average) \\
\hline
\end{tabular}

$\mathrm{MED}=$ morphine equivalent dose.

A tapering plan was prepared for each patient, according to individual needs. Opioid doses were tapered at a rate suitable for the patient, usually $10 \%$ every $1-2$ weeks.

Psychological support was provided via ZENDOSE, a validated web-based mental health and wellness tool [14]. ZENDOSE uses such evidence-based techniques as CBT, Dialectical Behavior Therapy (DBT), ACT, mindfulness and relaxation. It offers patient education materials relevant to living with chronic pain. ZENDOSE provides customized mental health care plans targeting patient's unique difficulties using brief assessments. Patients can record their goals and set reminders on the site. Participants used a daily log on ZENDOSE to track relevant aspects of their medical, physical, and mental well-being (e.g. blood pressure, glucose levels, appointments, sleep, mood). The ZENDOSE Twitter feed suggests a topic related to improving quality of life each day; participants were encouraged to spend 15-30 minutes daily in meditative reflection.

Medical cannabis provided pharmacological support throughout the tapering process. Authorizations for cannabis specified cannabidiol (CBD) and THC in the range of 4-6\%. Doses related directly to the opioid taper: $0.5 \mathrm{~g} /$ day for each $10 \%$ reduction in opioid dose, as needed. Use of cannabis was restricted to sublingual, oral or inhalation by vaporization (vaping) routes.

Physicians monitored patients regularly at intervals suited to the individual patient's needs, typically every 1-2 weeks. At each visit, the patient's pain, sleep, function, quality of life, benefits of tapering, use of non-pharmacological therapies, effects of medical cannabis, opioid risk, and opioid withdrawal symptoms were assessed.

Data on patient wellness, opioid doses and medical cannabis use were collected at each visit and after six months.

\section{Results}

Six months after beginning MCORP, 156 patients $(26 \%)$ had stopped using opioids altogether. An additional 329 patients $(55 \%)$ had reduced their opioid use by an average of $30 \%$. Cannabis use among these patients ranged from $1-3 \mathrm{~g}$ per day.

There was no change in the opioid use of 114 patients $(19 \%)$ - neither reduction nor escalation in opioid dose. One patient with poorly controlled pain and an aggravated pain condition increased opioid intake.

With that one exception, all patients expressed satisfaction with their pain control, sleep and quality of life. No opioid withdrawal symptoms were noted in follow-up appointments.

\section{Discussion}

MCORP was very helpful to many patients, but its implementation comes with several cautions. Not all patients are appropriate candidates for cannabis therapy: those under 25 years of age; those with a personal or strong family history of psychosis; patients who are pregnant, breastfeeding or planning a pregnancy; and patients with an active substance use disorder, cardiovascular, respiratory, severe liver, or severe kidney disease. Caution should be exercised with patients with concurrent mood or anxiety disorders, those who smoke tobacco or drink alcohol heavily, those with risk factors for cardiovascular disease, and patients taking high doses of sedating medications [15].

For patients unfamiliar with cannabis, low starting doses are advised, with titration to effect. Selected routes of administration reduce potential for harm: vaporization, sub- 
lingual sprays or edible products. In vaping, cannabis vapor is inhaled over five seconds, held for 10 seconds, and slowly exhaled [15]. Cannabis vaporizes at a lower temperature than combustion, and the vapor is less noxious than its smoke. Vaping extracts cannabinoids more efficiently than smoking, and is amendable to self-titration. Ingestion provides a slower onset and longer duration of action [16]. Patients were advised to consume cannabis in a comfortable and familiar environment, and to wait a few minutes between inhalations, or 30-60 minutes after ingesting small amounts of cannabis, in order to assess its impact [16].

MCORP participants in this program consumed a moderate amount of cannabis (1-3g/day). This pilot study covered a period of six months. In a recent Dutch study, where $43 \%$ of medical marijuana users were simultaneously prescribed opioids, the average duration of cannabis use was 251 days [17].

Taper rates for each patient were established at the outset of the program. Plans typically reduced the amount of medication by $10 \%$ every $1-2$ weeks until the smallest available dosage unit was reached. At that point, time between reductions was doubled. Some flexibility was required, as determined during monitoring visits. Tapering was paused if there was a worsening of pain, mood or function, and in times of intense stress.

Participating patients were encouraged to continue any psychological support program that was part of their multimodal chronic pain therapy. Cognitive behavioral therapy is recommended during opioid tapering [5]. Mindfulness training is an effective self-management technique for multiple medical conditions, including pain [13, 18], depression [19] and anxiety [20] - all of concern during a tapering program. Such therapeutic approaches are often uninsured, and can be prohibitively expensive for many patients. This pilot study offered patients the validated, free access web-based program, ZENDOSE. Its log feature enabled both patients and healthcare providers to track progress through tapering.

\section{Conclusion}

It is incumbent on physicians to seek ways to reduce harm to patients. Those related to the use of opioids are serious, including death. This MCORP pilot study outlines a patientcentered approach, with an individualized program for tapering opioid use. The positive results justify further investigation.

\section{Conflicts of Interest}

The author declares that there is no conflict of interest.

\section{Acknowledgements}

No funding, equipment or materials were received in developing this program, or in the writing of this submission. The author declares no conflict of interest. The views expressed in this article are those of the author, and not an official position of the University of Toronto. The author thanks Marcia Darling for her assistance in drafting this manuscript.

\section{References}

[1] J. W. Busse, S. Craigie, D. N. Juurlink, A. Carrasco, E. Alk, T. Agoritsas et al. Appendix 1 (as supplied by the authors): The 2017 Canadian Guideline for Opioids for Chronic Non-Cancer Pain. Appendix to Canadian Medical Association Journal [Internet]. 2017 [cited 2018 Oct 23]. 189: E659-66 [105 pages]. doi: 10.1503/cmaj.170363. Available from: http://www.cmaj.ca/content/suppl/2017/05/03/189.18.E659.D C1.

[2] T. Gomes, S. Greaves, D. Martins, M. Tadrous, M. M. Mamdami, J. M. Paterson et al. Latest Trends in OpioidRelated Deaths in Ontario: 1991 to 2015 [Internet]. Toronto: Ontario Drug Policy Research Network; April 2017 [cited 2018 Oct 23]. Available from http://odprn.ca/wpcontent/uploads/2017/04/ODPRN-Report_Latest-trends-inopioid-related-deaths.pdf.

[3] J. O. Prochaska, C. C. DiClemente. The transtheoretical approach. In: Norcross, JC, Goldfried, MR, editors. Handbook of psychotherapy integration. 2nd ed. New York: Oxford University Press; 2005. p. 147-171.

[4] G. L. Zimmerman, C. G. Olsen, D. O. Bosworth. A 'Stages of Change' Approach to Helping Patients Change Behavior. American Family Physician 2000; 61 (5): 1409-1416.

[5] C. Berna, R. J. Kulich, J. P. Rathmell. Tapering Long-term Opioid Therapy in Chronic Noncancer Pain: Evidence and Recommendations for Everyday Practice. Mayo Clinic Proceedings 2015; $90 \quad$ (6): 828-42. doi: 10.1016/j.mayocp.2015.04.003.

[6] Michael G. DeGroote National Pain Centre. Canadian Guideline for Safe and Effective Use of Opioids for Chronic Non-Cancer Pain; Appendix B-12: Opioid Tapering [Internet]. 2010 [cited 2018 Oct 18]. Available from: http://nationalpaincentre.mcmaster.ca/opioid_2010/cgop_b_ap p_b12.html.

[7] J. Elikkottil, P. Gupta, K. Gupta. The analgesic potential of cannabinoids. Journal of Opioid Management 2009 Nov-Dec; 5 (6): $341-57$.

[8] K. F. Boehnke, E. Litinas, D. J. Clauw. Medical Cannabis Use Is Associated With Decreased Opiate Medication Use in a Retrospective Cross-Sectional Survey of Patients With Chronic Pain. The Journal of Pain 2016; 17 (6): 739-44. doi: 10.1016/j.jpain.2016.03.002.

[9] L. Degenhardt, N. Lintzeris, G. Campbell, R. Bruno, M. Cohen, M. Farrell et al. Experience of adjunctive cannabis use for chronic non-cancer pain: findings from the Pain and Opioids IN Treatment (POINT) study. Drug and Alcohol Dependence 2015; 147: 144-50. doi: 10.1016/j.drugalcdep.2014.11.031.

[10] M. A. Ware, T. Wang, S. Shapiro, A. Robinson, T. Ducret, T. Huynh et al. Smoked cannabis for chronic neuropathic pain: a randomized controlled trial. Canadian Medical Association Journal 2010; 182 (14): E694-E701. 
[11] D. I. Abrams, P. Couey, S. B. Shade, M. E. Kelly, N. L. Benowitz. Cannabinoid-Opioid Interaction in Chronic Pain. Clinical Pharmacology and Therepeutics 2011; 90 (6): 844891.

[12] P. Lucas, Z. Walsh. Medical cannabis access, use, and substitution for prescription opioids and other substances: A survey of authorized medical cannabis patients. The International Journal on Drug Policy 2017; 42: 30-35. doi: 10.1016/j.drugpo.2017.01.011. Epub 2017 Feb 9.

[13] F. Zeidan, N. S. Gordon, J. Merchant, P. Goolkasian. The effects of brief mindfulness meditation training on experimentally induced pain. The Journal of Pain 2010; 11 (3): 199-209.

[14] K. Rod. Finding Ways to Lift Barriers to Care for Chronic Pain Patients: Outcomes of Using Internet-Based SelfManagement Activities to Reduce Pain and Improve Quality of Life. Pain Research and Management 2016; 2016: 8714785. doi: 10.1155/2016/8714785. Epub 2016 Mar 1.

[15] College of Family Physicians of Canada. Authorizing Dried Cannabis for Chronic Pain or Anxiety: Preliminary Guidance from the College of Family Physicians of Canada. Mississauga, ON: College of Family Physicians of Canada [Internet]. 2014 [cited 2018 Oct 23]. Available from: https://www.cfpc.ca/uploadedFiles/Resources/ PDFs/Authoriz ing\%20Dried\%20Cannabis\%20for\%20Chronic\%20Pain\%20o r\%20Anxiety.pdf.
[16] Health Canada. Information for Health Care Professionals: Cannabis (marihuana, marijuana) and the cannabinoids [Internet]. 2013 [cited 2018 Oct 23]. Available from: http://www.hc-sc.gc.ca/dhpmps/alt_formats/pdf/marihuana/med/infoprof-eng.pdf.

[17] A. Hazekamp, E. R. Heerdink. The prevalence and incidence of medicinal cannabis on prescription in The Netherlands. European Journal of Clinical Pharmacology 2013; 69 (8): 1575-1580.

[18] E. L. Cassidy, R. J. Atherton, N. Robertson, D. A. Walsh, R. Gillett. Mindfulness, functioning and catastrophizing after multidisciplinary pain management for chronic low back pain. Pain 2012; 153 (3): 644-650.

[19] K. Hopkins, P. Crosland, N. Elliott, S. Bewley, Clinical Guidelines Update Committee B. Diagnosis and management of depression in children and young people: summary of updated NICE guidance. British Medical Journal 2015; 350: h824. doi: 10.1136/bmj.h824.

[20] B. Smith, K. Metzker, R. Waite, P, Gerrity. Short-form mindfulness-based stress reduction reduces anxiety and improves health-related quality of life in an inner-city population. Holistic Nursing Practice 2015; 29 (2): 70-77. doi: 10.1097/HNP.0000000000000075. 